\title{
Growth, Microhardness and Electrical Properties of L- Histidine Hydrochloride Monohydrate Nonlinear Optical Single Crystal
}

\author{
P.Koteeswari \\ Department of Physics \\ Madha Engineering College \\ Chennai
}

\author{
S.Suresh* \\ Department of Physics \\ Loyola College, Chennai
}

\author{
P.Mani \\ Department of Physics \\ Hindustan Institute of \\ Technology, Chennai
}

\begin{abstract}
Single crystal of 1-histidine hydrochloride monohydrate (LHHC) was successfully grown by slow evaporation technique. The cell parameters and the crystallinity of the grown crystal were estimated by the single crystal. Mechanical strength of the grown crystal was analyzed using Vickers microhardness tester. Nonlinear optical property of the crystal was confirmed by Kurtz and Perry powder technique. AC conductivity and photoconductivity experiments are also carried out and the results are discussed.
\end{abstract}

\section{Keywords}

Crystal growth from solution, Single XRD, Microhardness, SHG, AC conductivity and Photoconductivity studies.

\section{INTRODUCTION}

Organic nonlinear optical materials have been investigated due to their potentially high nonlinearities and rapid response in electro-optic effect compared to inorganic NLO materials. These molecular organic compounds with one or more aromatic systems in conjugated positions, leading to charge transfer systems have been intensely studied for the past two decades [1]. Considerable theoretical and experimental investigations have been done in order to understand the microscopic origin of nonlinear behavior of organic NLO materials $[2,3]$. The conjugated $\pi$ electron system provides a pathway for the entire length of conjugation under the perturbation of an external electric field. Fictionalization of both ends of the $\pi$ conjugated system with appropriate electron donor and acceptor groups can increase the asymmetric electronic distribution in either or both the ground and excited states, thus leading to an increased optical nonlinearity [4]. When acceptor and donor moieties are placed at terminal position of conjugated backbone, both linear and nonlinear optical properties have increased significantly which involves correlated and high delocalized $\pi$ electron states. The magnitudes molecular polarisability and hyperpolarisability coefficients are found to increase super linearly with an increase in conjucation length between the donor and the acceptor. The strength of donor and acceptor groups and order of their stacking along the backbone plays important roles in determining the magnitude of nonlinear optical (NLO) efficiency [5]. In semi-organics, polarizable organic molecules are stoichiometrically bound within an organic host [6]. In recent years, the NLO properties of semiorganic complex products have attracted great interest because these metal-organic complexes combine the high optical nonlinearity and chemical flexibility of organics with physical ruggedness of inorganics [7, 8]. Nowadays amino acids are noticeable materials due to its component nonlinear optical efficiency. Some complexes of amino acids with inorganic salts have already been reported by many researchers $[9,10]$, and that has proven to be a good candidate for nonlinear optical applications. In the present investigation, the growth aspects of the LHHC crystal have been carried out by slow evaporation technique. The grown crystal has been subjected to XRD, Microhardness, SHG, AC conductivity and photoconductivity studies. The results of these investigations are discussed.

\section{EXPERIMENTAL}

The L-histidine and hydrochloric acid were taken in equimolar ratio in double distilled water to prepare the saturated solution of L-histidine hydrochloride mono hydrate (LHHC). The solution obtained is stirred well at room temperature using a temperature controlled magnetic stirrer to yield a homogenous mixture of solution. Then the solution is filtered using a Whatmann filter paper and was allowed to evaporate at room temperature. The solution is recrystallized several times in order to increase the purity of the crystal. Optically clear and good quality seed crystal is kept inside the purified saturated solution and the solution is allowed to evaporate at room temperature, which produces an improved optically high quality within a period of 30 days. The photograph of the as grown single crystal is shown in Fig.1

\section{RESULTS AND DISCUSSION}

\subsection{Single-crystal X-ray diffraction}

Single crystal $\mathrm{X}$-ray diffraction analysis was carried out to determine the lattice parameters. The grown crystals have orthorhombic structure with $\mathrm{P} 2{ }_{1} 2_{1} 2_{1}$ space group. The lattice parameter values of the grown crystals are $a=6.82 \AA$, $b=8.91 \AA$, $c=15.286 \AA$. The single crystal data are in good agreement with reported values [11]. 


\subsection{Microhardness study}

The mechanical properties of crystals are evaluated by mechanical testing which reveals certain mechanical characteristics. The fastest and simplest type of mechanical testing is hardness measurement. Among the different testing methods, the Vickers hardness test is more commonly used. Microhardness study was conducted using a Vickers microhardness tester fitted with a diamond pyramidal indentor. Microhardness measurements were made using a Leitz microhardness tester fitted with a diamond pyramidal indentor. Single crystals of LHHC were subjected to microhardness studies. The applied load was varied from 10 to $50 \mathrm{~g}$ for a constant indentation period of $10 \mathrm{~s}$. The Vicker'shardnessnumber Hv is calculated using the relation

$$
H_{V}=1.8544 P / d^{2} \mathrm{~kg} / \mathrm{mm}^{2}
$$

where $\mathrm{P}$ is the indent or load in $\mathrm{kg}$ and $\mathrm{d}$ is the diagonal length of the impression in $\mathrm{mm}$. The variation of $\mathrm{Hv}$ with applied load is shown in Fig.2. It is evident from the plot that the microhardness of the crystal decreases with increasing load. The decrease in the microhardness values of LHHC with increasing load is in agreement with the normal indentation size effect (ISE).

\subsection{SHG efficiency test}

The SHG efficiency of LHHC has been found by Kurtz and Perry powder technique. The powder sample prepared from the grown crystals has been subjected to this test. The second harmonic output has been generated by irradiating the powder samples by a pulsed laser beam of Nd: YAG laser with a pulse width of 8 ns. The efficiency of the energy (frequency) conversion is confirmed by the emission of green light from the powder sample. For a laser input pulse of $6.2 \mathrm{~mJ}$, the second harmonic signal $(532 \mathrm{~nm})$ of $88.42 \mathrm{~mW}$ and 272.10 $\mathrm{mW}$ respectively were obtained through KDP and LHHC single crystal. KDP sample has been used as the reference material and output power intensity of LHHC has been found to be three times as that of output power intensity of KDP. This result agrees well with the literature value [11].

\subsection{Electrical AC conductivity studies}

The $a c$ conductivity measurements are taken using HIOKI 3532-50 LCR HITESTER in the frequency range $50 \mathrm{~Hz}$ to 5 MHz. Temperature dependence of conductivity is shown in Fig. 3. The Arrhenius plot of $\sigma \mathrm{T}$ versus $1000 / \mathrm{T}$ is shown in Fig.4. It is evident from the graph that the conductivity increases with the temperature. Accordingly the value of activation energy for ionic migration is estimated from the slope of the Arrhenius plot. The line of best fit for the plot of $\log \sigma \mathrm{T}$ versus $1 / \mathrm{T}$ obeys Arrhenius relationship.

$$
\sigma=\sigma_{0} \exp \left(E_{a} / k T\right)
$$

Where $\sigma_{0}$ is the pre-exponent factor, $\mathrm{E}_{\mathrm{a}}$ the activation energy for the conduction process and $\mathrm{k}$ is the Boltzmann constant. Therefore, the sample exhibits Arrhenius type conductivity behavior in the frequency range of investigation. The activation energy of LHHC for the conduction process, calculated from the plot is found to be $0.060 \mathrm{eV}$. The conduction mechanism can be explained by the rotation of ions when the temperature of the material is increased. The rotation of ions gives local displacement of electrons in the direction of the applied field, which in turn gives rise to induced polarization when the material is subject to intense laser beam. The lower value of activation energy $(0.060 \mathrm{eV})$ predicted in the present investigation suggests that the crystal is free from defects. When the crystal is free from defects, the hydrogen bond interactions will not be weakened.

\section{Photoconductivity Studies}

Photoconductivity measurements were made using Keithley 485 picoammeter. The dark current was recorded by keeping the sample unexposed to any radiation. Fig.5 shows the variation of both dark current (Id) and photocurrent (Ip) with applied field. It is seen from the plots that both Ip and Id of the sample increase linearly with applied field. It is observed from the plot that the Photo current is always higher than the dark current, thus confirming the positive photoconductivity [12].

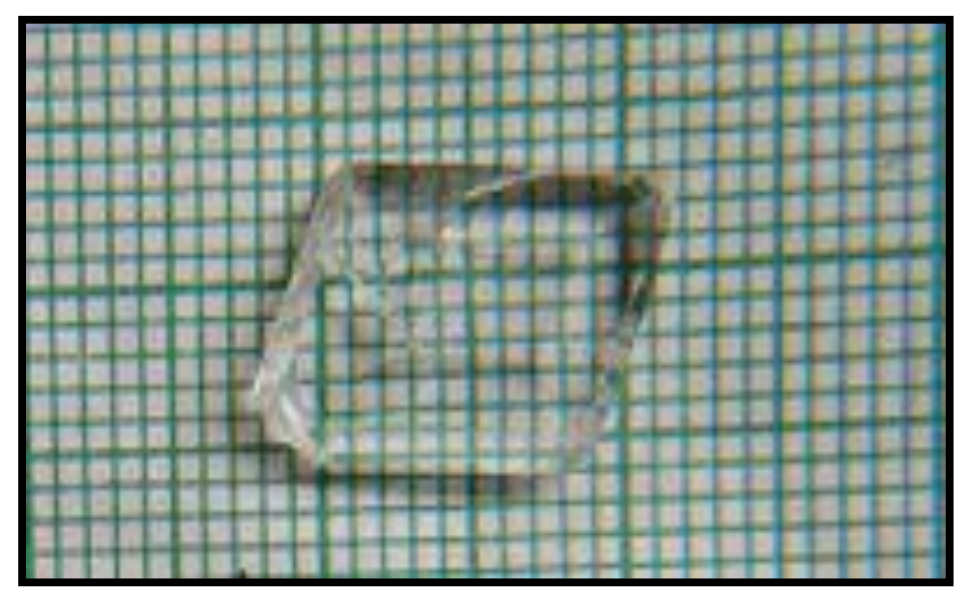

Fig.1. Photograph of as grown crystal 


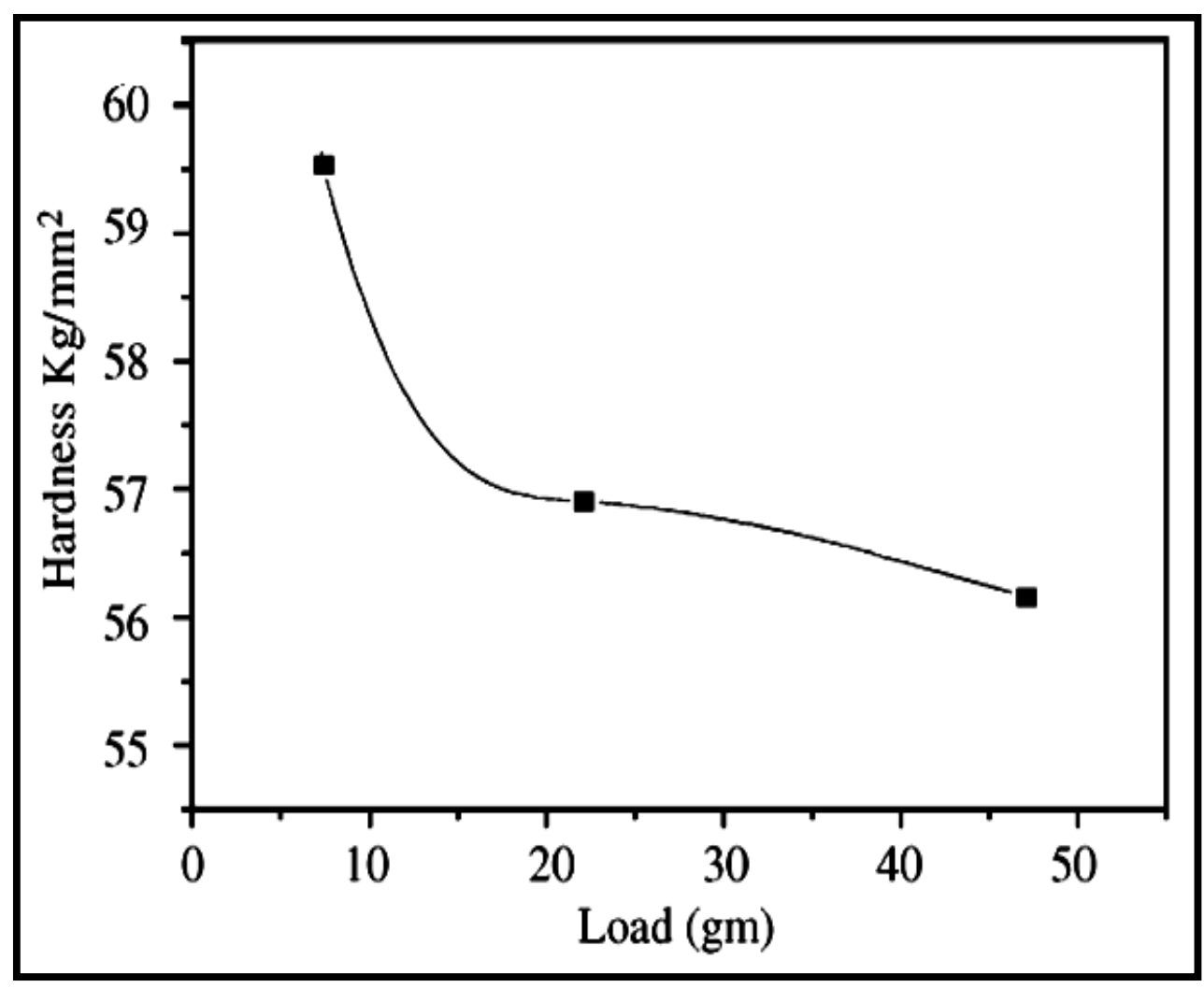

Fig.2. Plot of Load vs Hardness number

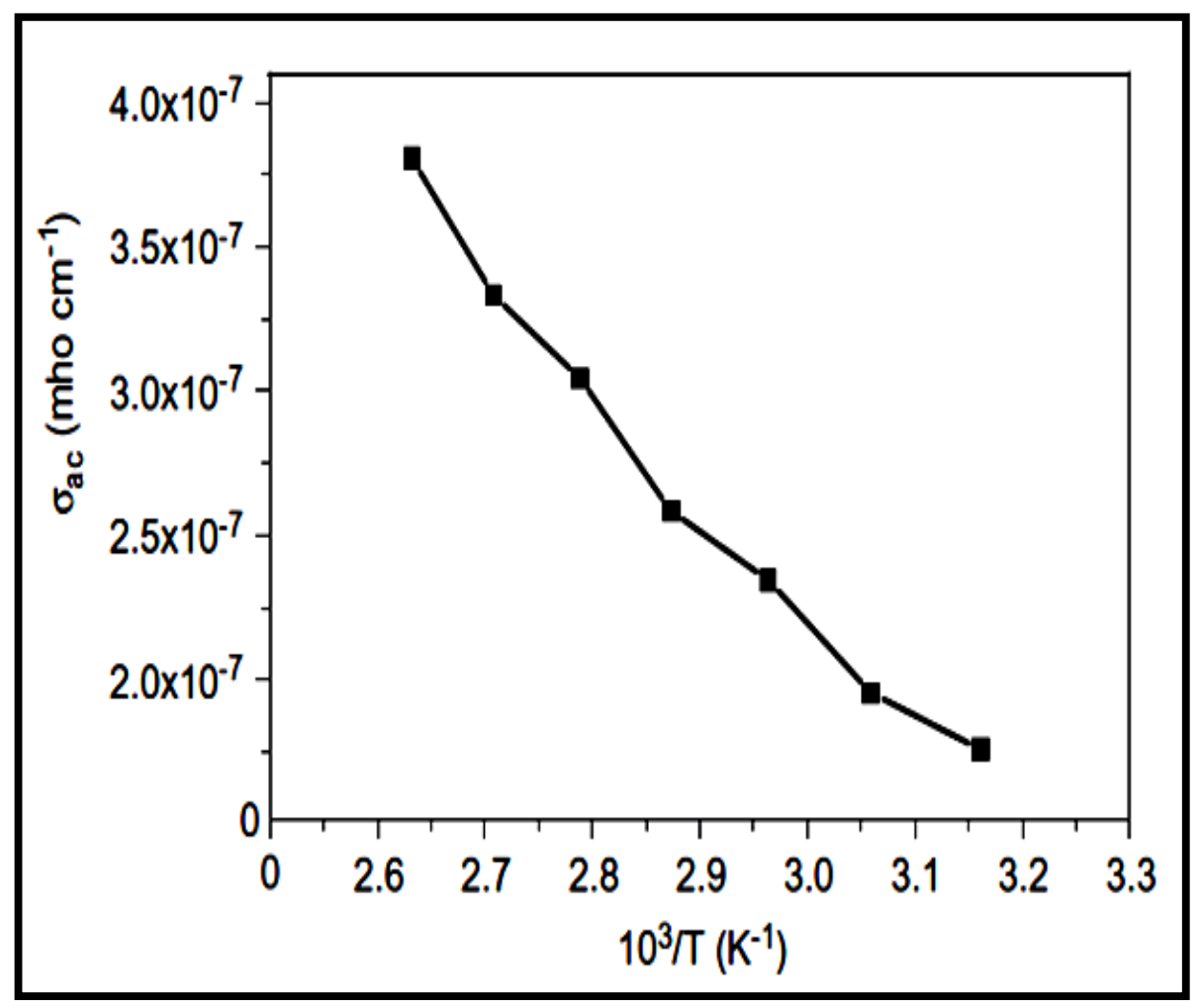

Fig. 3. Variation of ac conductivity with 1000/T 


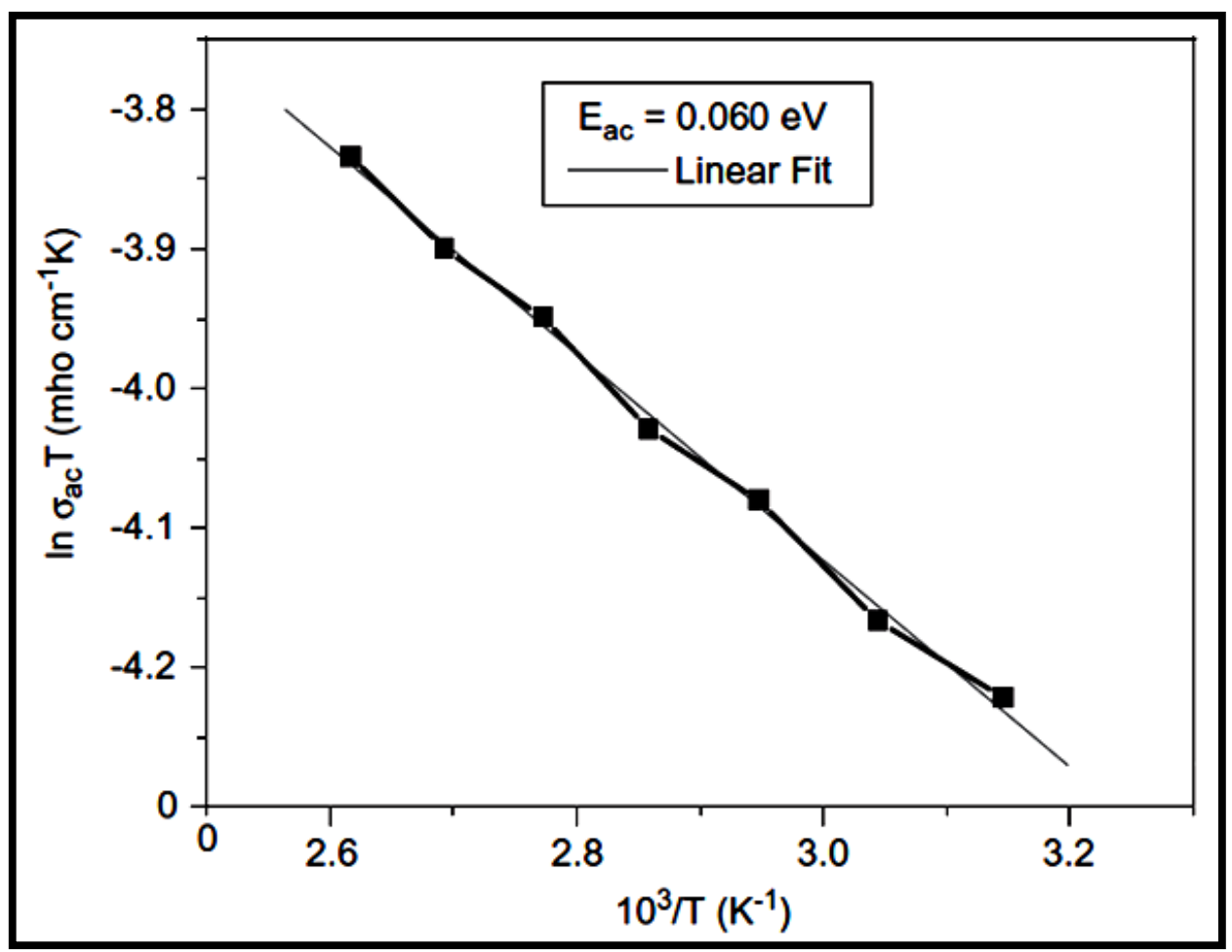

Fig.4. Plot of $\ln \left(\sigma_{\mathrm{ac}}\right) \mathrm{vs} 1000 / \mathrm{T}$

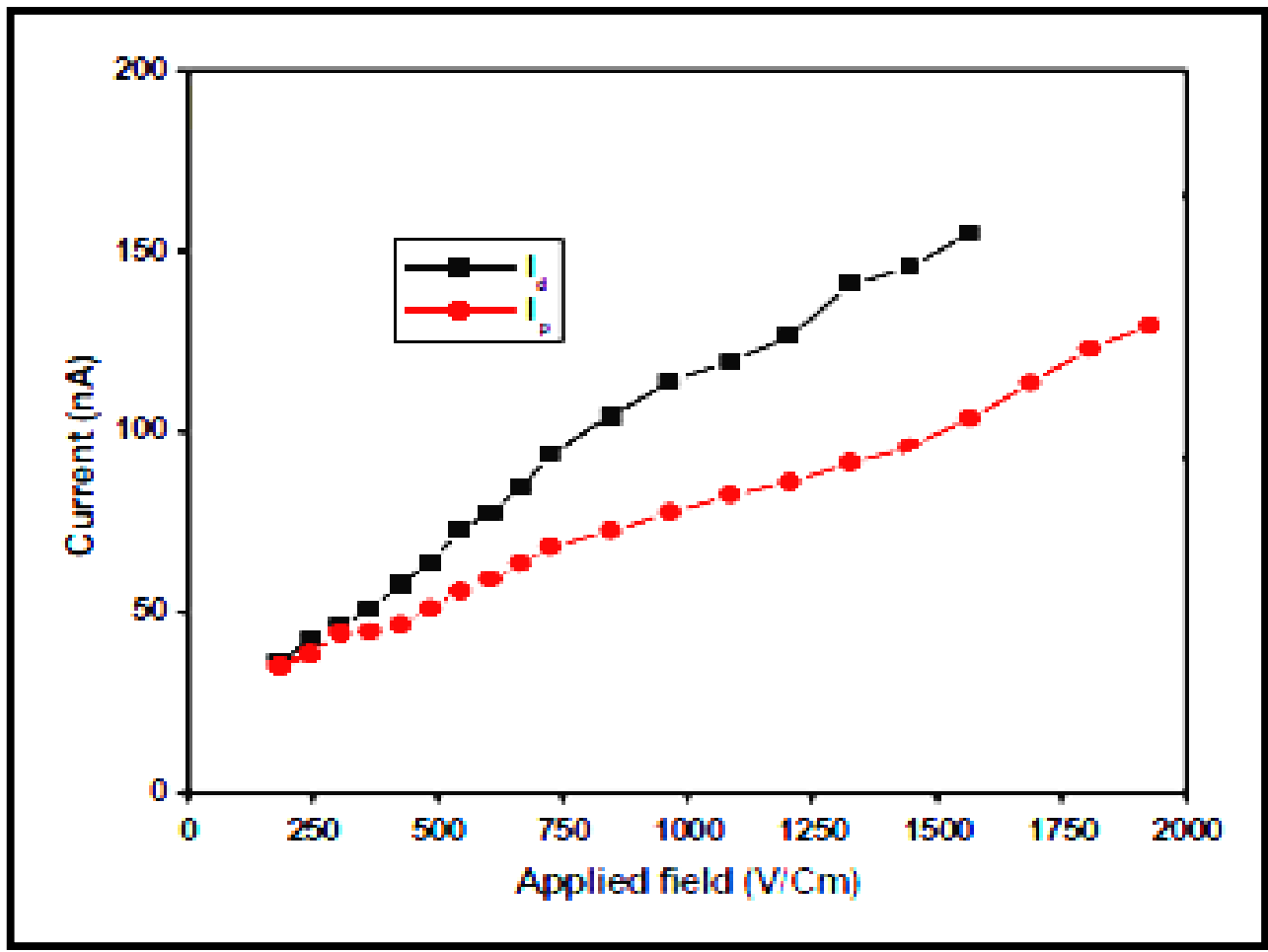

Fig.5. Plot of field dependent photoconductivity of the LHHC single crystal 


\section{CONCLUSION}

Single crystal of semi-organic LHHC was grown from aqueous solution by a slow evaporation technique. Single crystal X-ray diffraction studies confirm that the grown crystal belongs to orthorhombic crystal system with space group $P 2_{1} 2_{1} 2_{1}$. The single crystalline nature is thus confirmed and the space group reveals that the material shows no inversion symmetry (non-centrosymmetric). XRD analysis fulfills one of the requirements for the material to exhibit NLO property. Mechanical behaviour has been studied by Vickers microharness test. The hardness studies show the decreasing nature of hardness number with increase in load. . Mechanical properties were analysed to get an idea about the laser damage threshold.The studies on the NLO property confirmed the second harmonic conversion efficiency of the crystal has been found to be comparable to many NLO crystals and better than KDP. The activation energy is determined from the plots of AC conductivity. Electrical conductivity analysis reveals the delocalization of charges for the material to generate Second Harmonic frequency (SHG). The conductivity is found to increase with the increase of applied field. The generation of dipoles is also confirmed from the positive photoconductivity nature of the material. Photoconductivity study reveals positive photoconductive nature of the crystal. These studies were carried out to confirm noncentrosymmetric nature, polarization mechanism and mechanical strength of the material relevant to NLO behavior.

\section{REFERENCES}

[1] Goto Y., Hayashi A., Kimura Y. and Nakayama M., 'Second harmonic generation and crystal growth of substituted thienyl chalcone', J. Cryst. Growth, Vol. 108, (1991) pp. 688-698.

[2] Kerkoc P., Zgonik M., Sutter K., Bosshard C. and Gunter P., J. Opt. Soc. Am., Vol. B7, (1990) pp. 313-319

[3] Dimitriev V.G., Gurzadyan G.G. and Nikogosyan D.N., 'Handbook of Nonlinear Optical Crystals', Springer, Berlin (1991)

[4] Prasad P.N. and Williams D.J. (1991), 'Introduction to Nonlinear Optical Effects in Molecules and Polymers', Wiley, New York (1991)

[5] Blanchard-Desce M., Runser C., Fort A., Barzoukas M., Lehn J.M., Bloy V. and Alain V, Chem. Phys., Vol. 199(1995) pp. 253-261

[6] Y.J. Ding, X. Mu, X.J. Gu, Nonlinear Opt. Phys.Mater. $9(2000) 21$

[7] P.R. Newman, L.F. Warren, P. Cunningham, T.Y. Chang, D.E. Cooper, G.L. Burdge, P. Polok Dingels, C. K. Lowe-Ma, Mater.Res. Soc. Proc. 173(1990)557.

[8] V. Venkataraman, G. Dhanraj, V.K. Wadhawan, J.N. Sherwood, H.L. Bhat, J. Cryst. Growth, 154(1995)92.

[9] S. Prahadeeswaran, H.L. Bhat, N.S. Kini, A.M.Umarji, P. Balaya, P.S. Goyal, J. Appl. Phys. 88(2000)5935

[10] S. Mukerji, T. Kar, Metall. Mater. Trans. 31(2000)3087

[11] J. Madhavan, S. Aruna, P.C. Thomas, M. Vimalan, S.A. Rajasekar, P. Sagayaraj, Cryst. Res. Technol. 42 (2007) 59.

[12] V. N. Joshi, Photoconductivity, Marcel Dekker, New York (1990) 